\title{
Non-traumatic Tension Gastrothorax: A Potential Mimicker of Tension Pneumothorax
}

\author{
Jonathan D Pierce ${ }^{1 *}$, Neal R Shah ${ }^{1}$, Ata A Rahnemai-Azar ${ }^{1}$, Amit Gupta $^{1}$ \\ 1. Department of Radiology, University Hospitals Cleveland Medical Center/Case Western Reserve University School of Medicine, \\ Cleveland, $\mathrm{OH}, \mathrm{USA}$ \\ * Correspondence: Jonathan D. Pierce, MD, Department of Radiology, University Hospitals Cleveland Medical Center, 11100 Euclid \\ Ave, Cleveland, OH 44106, USA \\ (₫Jonathan.Pierce@uhhospitals.org)
}

Radiology Case. 2021 Aug; 15(8):1-7 :: DOI: 10.3941/jrcr.v15i8.4144

\begin{abstract}
Tension gastrothorax is a rare, life-threatening clinical condition caused by intrathoracic herniation of the stomach through a diaphragmatic defect which becomes increasingly distended over time. If not recognized promptly, this can rapidly progress to respiratory distress, mediastinal shift, and hemodynamic compromise. Initial clinical presentation and imaging findings closely mirror those of tension pneumothorax, confounding diagnosis and potentially leading to unnecessary interventions with increased risk of morbidity and mortality. Here, we present a case of an elderly female who presented with a non-traumatic tension gastrothorax and a review of key imaging features and strategies to aid in recognition and accurate diagnosis of this emergent clinical entity.
\end{abstract}

\section{CASE REPORT}

\section{CASE REPORT}

An 87-year-old female with a past medical history of chronic obstructive pulmonary disease requiring continuous oxygen supplementation and atrioventricular nodal reentrant tachycardia presented to the emergency department with dyspnea and tachycardia. On arrival to the emergency department, the patient was found to be tachycardic with an initial heart rate of 204 beats per minute (bpm). Additionally, her initial blood pressure was 105/80 millimeters of mercury $(\mathrm{mmHg})$ however, she quickly became progressively hypotensive $(80 / 50 \mathrm{mmHg})$ prompting volume resuscitation, pressor support, and urgent electrical cardioversion. Once hemodynamic stability was achieved, a thorough physical exam demonstrated diminished breath sounds bilaterally, greater on the left side.
A portable supine radiograph of the chest was performed which revealed a large lucent intrathoracic structure with absence of the normal gastric air bubble and left lung parenchyma as well as significant rightward mediastinal shift, raising concern for possible tension pneumothorax. (Figure 1). However, upon review of prior imaging in our picture archiving and communication system (PACS), it was noted that the patient had a large preexisting left-sided diaphragmatic hiatal hernia seen on a computed tomography (CT) scan from several years prior (Figure 2). After discussion of these findings with the ordering clinician, this prompted further assessment with a CT scan of the chest rather than empirical treatment with chest tube placement. Meanwhile, a bedside ultrasound was also performed by the ordering clinician which identified left pleural sliding, a finding which contradicted the diagnosis of pneumothorax. 
The subsequently performed $\mathrm{CT}$ of the chest revealed a significant amount of air within the left hemithorax with near complete collapse of the left lung and contralateral mediastinal shift, again, seemingly indistinguishable from a large tension pneumothorax (Figure 3A). However, upon further inspection using multiplanar reconstructed oblique-coronal images, it was determined that the left hemithoracic air was in fact contiguous with a bowel loop (Figure 3B-C), which could be traced inferiorly as duodenum. Therefore, the diagnosis of tension gastrothorax was made. Following nasogastric tube insertion, prompt decompression of the dilated stomach was performed and repeat radiograph of the chest showed marked improvement in the dilated intrathoracic stomach with associated improvement in aeration of the left lung (Figure 4). The patient tolerated the procedure well and was transferred to the Intensive Care Unit in stable condition for observation. Following evaluation by Thoracic surgery, it was determined that the patient's underlying cardiopulmonary comorbidities ultimately precluded her from undergoing definitive surgical repair, and she was subsequently discharged with initiation of palliative care.

\section{DISCUSSION}

\section{Etiology \& Demographics:}

Tension gastrothorax, first described by Ordog et al. in 1984, refers to a clinical condition in which a herniated intrathoracic stomach becomes significantly distended by trapped air, leading to respiratory distress, mediastinal shift, and hemodynamic compromise [1-3]. The presence of a diaphragmatic defect serves as a prerequisite for the condition and thus, cases are typically seen in the pediatric population with congenital diaphragmatic defects or later in life with acquired defects, most commonly in the setting of trauma. However, cases involving enlarging hiatal hernias as well as secondary to post-surgical complications have also been reported [4-8]. While the incidence of congenital diaphragmatic hernia is approximately 1 per 2,500-5000 live births, true tension gastrothorax is exceedingly rare and possesses a slight female predominance. Mechanistically, gastrothorax develops when increased intraabdominal pressure (i.e. blunt trauma, Valsalva maneuver, etc.) forces the stomach into the thorax through the diaphragmatic defect. Accumulation of gastric contents such as air, fluid, or food into the abnormally positioned and angulated gastroesophageal junction functions as a one-way valve, leading to increased intrathoracic pressure [2-4].

\section{Clinical \& Imaging Findings}

Patients with tension gastrothorax typically present with clinical symptoms indistinguishable from tension pneumothorax including acute dyspnea, chest pain, and tachycardia. If unrecognized, the enlarging intrathoracic stomach causes progressive mediastinal shift that can lead to respiratory failure, obstructive shock, and cardiac arrest. In addition to its mechanistic similarities, there is also significant overlap in radiographic findings to that of tension pneumothorax, making this entity particularly challenging to diagnose.
Correct interpretation of emergent imaging findings plays a critical role in differentiating the two entities. Chest radiographs of tension gastrothorax demonstrate a large airfilled lucent structure occupying the left hemithorax with or without air-fluid level [2]. There is associated compression of the ipsilateral lung and significant contralateral mediastinal shift. Several key radiographic features are useful for distinguishing a gastrothorax from a tension pneumothorax. These include: lack of the normally seen gastric bubble, poorly defined left hemidiaphragm (which is typically inferiorly displaced and readily identified in cases of tension pneumothorax), and/or the presence of an air-fluid level within the left hemithorax [2,4]. The diagnosis of tension gastrothorax is best evaluated on cross-sectional imaging such as CT with identification of communication between the distal-most portion of the intrathoracic stomach and bowel. However, the feasibility of performing CT imaging is largely dictated by the patient's hemodynamic stability and may not be an option in some emergent situations. Given this, several other imaging strategies are important for clinicians to keep in mind when approaching this diagnosis.

First and foremost, prior imaging studies are an invaluable resource that should always be reviewed and scrutinized when available. As seen in the case presented above, knowledge of a pre-existing diaphragmatic hernia seen on our patient's prior imaging was crucial in raising our clinical suspicion of an entity other than pneumothorax. Second, in cases where there is question regarding the certainty of a particular diagnosis, Radiologists should have a low threshold to recommend further evaluation with additional imaging and/or discuss their concerns with the ordering clinician as hasty insertion of a chest tube in misdiagnosed cases can lead to viscous or vascular injury with potentially catastrophic consequences [6, 8]. Lastly, while CT imaging is considered the gold standard for pneumothorax diagnosis, thoracic ultrasound has been shown to be more sensitive than traditional chest radiograph and has the added advantage of being available for use at point-of-care, making it a particularly useful imaging modality for troubleshooting in equivocal cases [9]. Visualization of pleural sliding on lung ultrasound corresponding to the movement of the visceral pleura on the parietal pleura during respiration is the most important sonographic finding in normal aerated lung and effectively rules out the diagnosis of pneumothorax. This was performed by the ordering provider prior to discussion of the CT results of our case, which also pointed towards an alternate etiology for the left hemithoracic hyperlucency.

\section{Treatment \& Prognosis}

Once the diagnosis of tension gastrothorax has been made, prompt decompression of the stomach via nasogastric tube is the recommended initial treatment followed by definitive surgical repair of the diaphragmatic defect, once stabilized [4]. If recognized and treated early, the prognosis is excellent, with complete return to baseline in most instances.

\section{Differential Diagnosis}

We discuss briefly the differential diagnoses of a lucent hemithorax with contralateral mediastinal shift on chest radiograph, as seen in this case. 


\section{Tension Pneumothorax}

The primary differential for this case is a tension pneumothorax as discussed extensively in this case.

\section{Congenital diaphragmatic hernia}

The other consideration for this presentation is a congenital diaphragmatic hernia, which as mentioned above, can serve as a precursor for tension gastrothorax. Initial radiographic findings of a congenital diaphragmatic hernia include an indistinct appearance of the diaphragm with partial/full opacification of the ipsilateral hemothorax (typically left-sided). Deviated radiopaque lines and tubes are often a helpful diagnostic clue. Unlike tension gastrothorax, in addition to an intrathoracic stomach, multiple bowel loops are often also visualized within the affected hemithorax. The timing of presentation is a key factor for differentiating between these two entities. Congenital diaphragmatic hernias are often diagnosed prenatally or shortly after birth while tension gatrothorax typically presents months to years later.

\section{TEACHING POINT}

Tension gastrothorax is a rare life-threatening clinical condition which presents with clinical and radiographic features that closely mirror that of tension pneumothorax making diagnosis particularly challenging. It is important that clinicians possess a systematic approach to initial imaging studies including diligent review of prior exams, knowledge of key radiographic features distinguishing gastrothorax from tension pneumothorax, and swift evaluation with additional imaging modalities such as CT and/or point-of-care thoracic ultrasound in order to promptly recognize and successfully manage this emergent condition.

\section{REFERENCES}

1. Ordog GJ, Wasserberger J, Balasubramaniam S. Tension gastrothorax complicating post-traumatic rupture of the diaphragm. Am. J. Emerg. Med. 1984; 2(3):219-21. PMID: 6518015

2. Horst M, Sacher P, Molz G, Willi UV, Meuli M. Tension gastrothorax. J. Pediatr. Surg. 2005;40(9):1500-1504. PMID: 16150360

3. Ng J, Rex D, Sudhakaran N, Okoye B, Mukhtar Z. Tension gastrothorax in children: introducing a management algorithm. J. Pediatr. Surg. 2013;48(7):1613-1617. PMID: 23895982

4. Bunya N, Sawamoto K, Uemura S, et al. How to manage tension gastrothorax: a case report of tension gastrothorax with multiple trauma due to traumatic diaphragmatic rupture. Int J Emerg Med. 2017;10(1):4. PMID: 28127711

5. Næss P, Wiborg J, Kjellevold K, Gaarder C. Tension gastrothorax: acute life-threatening manifestation of late onset congenital diaphragmatic hernia $(\mathrm{CDH})$ in children. Scand. J. Trauma. Resusc. Emerg. Med. 2015; 23:49. PMID: 26104782

6. Singh, SP, Sukesan S, Kiran U, and Makhija N. Gastrothorax or tension pneumothorax: a diagnostic dilemma. J. Emerg. Trauma Shock. 2011; 4:128-129. PMID: 21633581

7. Shoji T, Tarui T, Kaita Y, Miyauchi H, Fukushima H, Yamaguchi Y. A survival case of tension gastrothorax due to hiatal hernia, the key of life-saving is thoracotomy. Am J Emerg Med. 2017; 35(1):199.e3-199.e5. PMID: 27503062

8. Gandhi S, Bhandarwar A, Sadhwani N, Patel C, Wagh A, Arora E. Combined laparoscopic and thoracoscopic approach for tension gastrothorax in a foramen of Bochdalek hernia. Int J Surg Case Rep. 2019;65:141-147. PMID: 31707302

9. Husain LF, Hagopian L, Wayman D, Baker WE, Carmody KA. Sonographic diagnosis of pneumothorax. J Emerg Trauma Shock. 2012;5(1):76-81. PMID: 22416161 


\section{FIGURES}

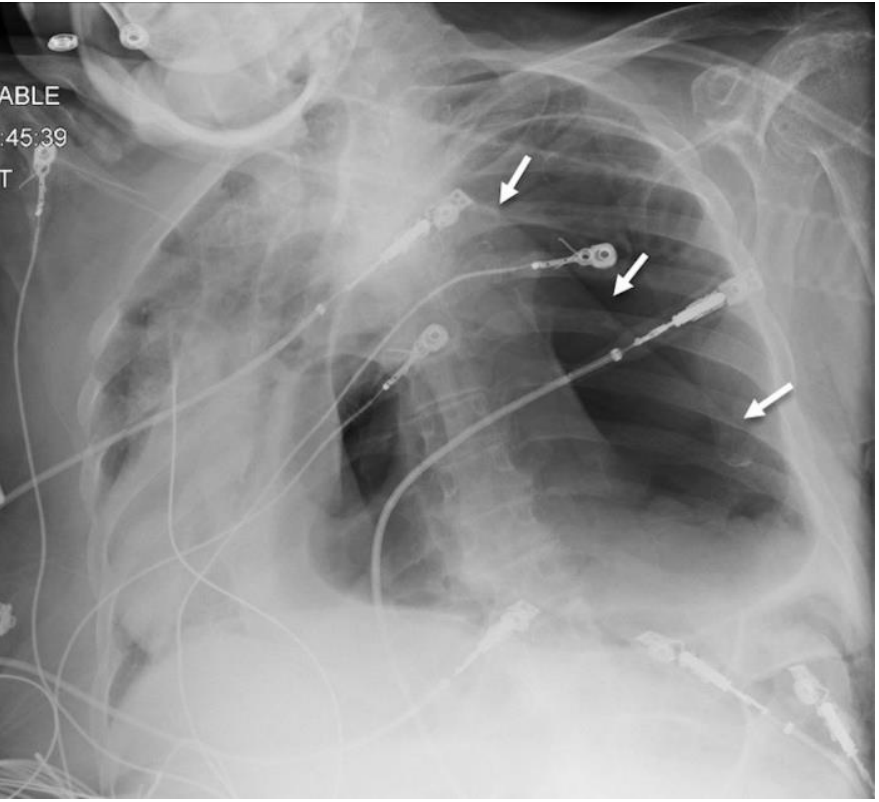

Figure 1 (left): An 87-year-old female with underlying diaphragmatic defect who presented with acute dyspnea and tachycardia secondary to tension gastrothorax.

Findings: Initial supine anteroposterior chest radiograph obtained shortly after arrival to the Emergency Department demonstrates a large central intrathoracic lucent structure (arrows) with absence of the normal gastric air bubble and left lung parenchyma as well as significant rightward mediastinal shift, raising concern for possible tension pneumothorax.

Technique: $85 \mathrm{kVp}$ x 2 mAs

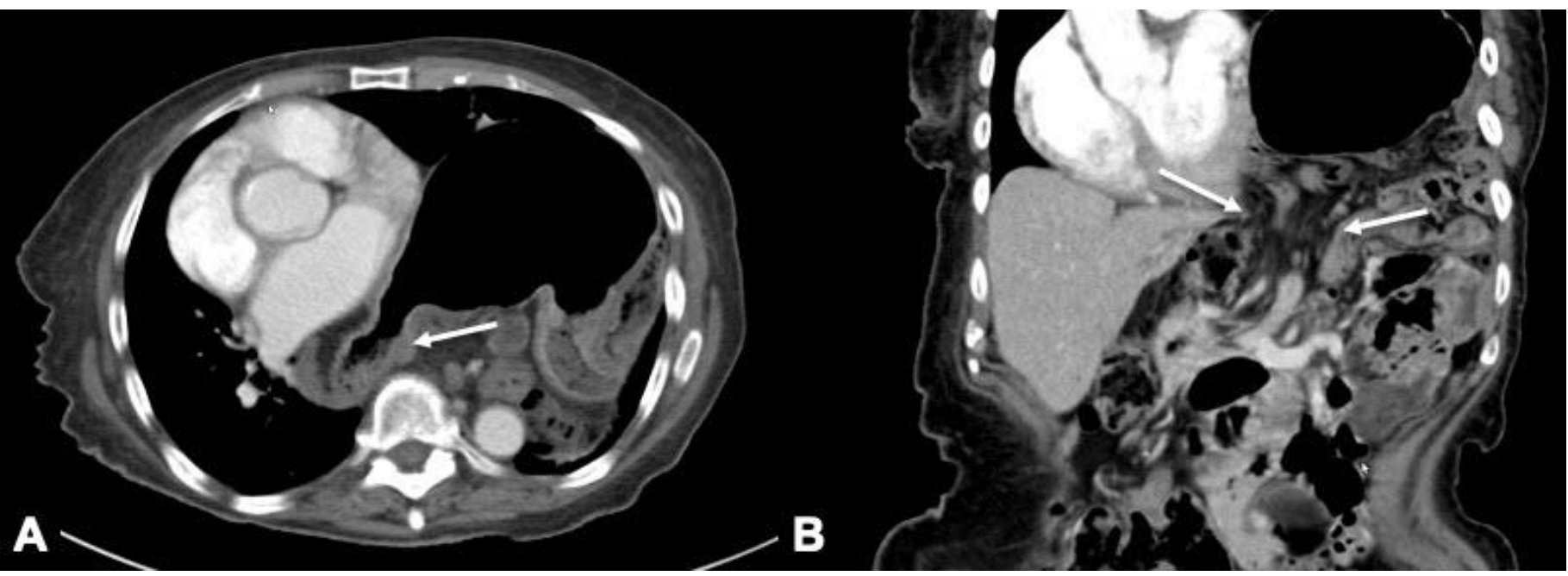

Figure 2: An 87-year-old female with underlying diaphragmatic defect who presented with acute dyspnea and tachycardia secondary to tension gastrothorax. Chest CT performed 3 years prior to patient's current symptoms.

Findings: (A) Remote contrast-enhanced axial and (B) coronal CT images of the lung bases/upper abdomen demonstrate a partially air-filled stomach and several loops of bowel within the left lung base which extend through a left-sided diaphragmatic defect (arrows in B).

Technique: Axial CT of the abdomen (A) with coronal reformatted image (B) following intravenous administration of $90 \mathrm{~mL}$ OptirayTM 350 (Ioversol injection 74\%) contrast material (Siemens SOMATOM Definition Flash, 5 mm slice thickness, 120 $\mathrm{kVp}$ x $304 \mathrm{mAs})$. 


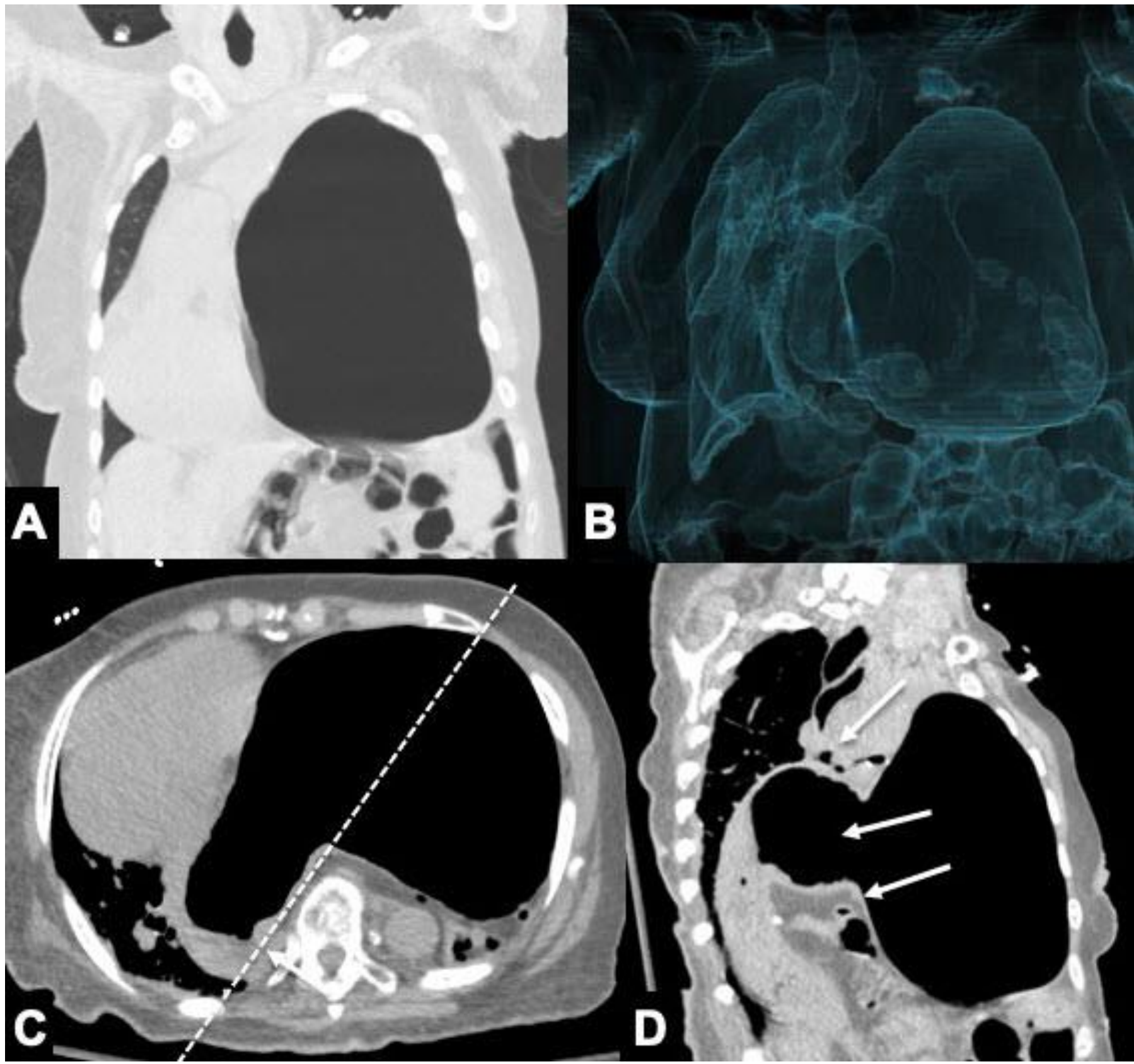

Figure 3: An 87-year-old female with underlying diaphragmatic defect who presented with acute dyspnea and tachycardia secondary to tension gastrothorax.

Findings: (A) Coronal CT image of chest in lung window setting and (B) 3-dimensional reconstructed image of the air-filled structures demonstrate a large, expansile air-filled structure within the left hemithorax with absence of lung parenchyma and significant contralateral mediastinal shift. However, careful inspection of axial (C) and oblique-coronal images (D, along the dotted line in C), reveals clear communication of the left-sided air with the bowel loop/duodenum (arrows in C and D), consistent with diagnosis of tension gastrothorax.

Technique: Non-contrast CT with coronal (A), 3-dimensional reconstruction of air-filled structures (B), axial (C), and obliquesagittal (D) reformats (Philips Brilliance iCT 256, $5 \mathrm{~mm}$ slice thickness, $100 \mathrm{kVp} \mathrm{x} 130 \mathrm{mAs}$ ). 


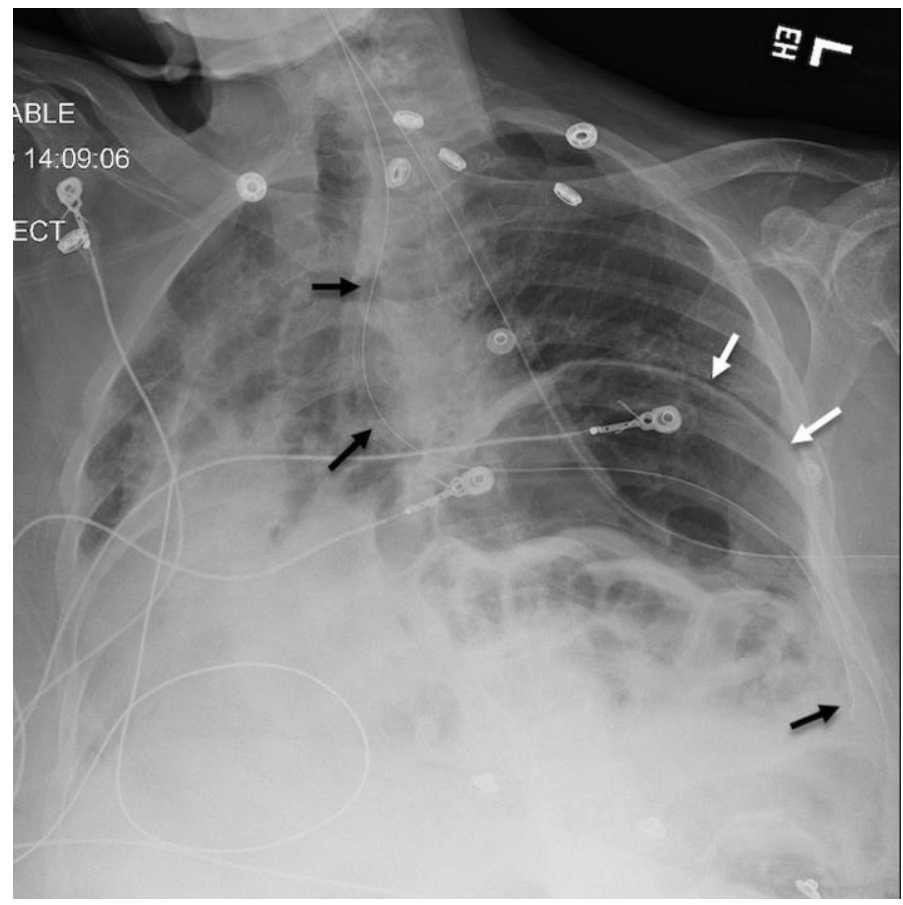

Figure 4 (left): An 87-year-old female with underlying diaphragmatic defect who presented with acute dyspnea and tachycardia secondary to tension gastrothorax.

Findings: Repeat supine anteroposterior chest radiograph following placement of a nasogastric tube (black arrows), and decompression of the stomach demonstrates significant interval improvement in dilatation of the stomach as well as improved aeration of the left lung. The residual gastric bubble, now below the level of the left hemidiaphragm, is annotated by white arrows.

Technique: $85 \mathrm{kVp}$ x 2 mAs

\begin{tabular}{|l|l|}
\hline Etiology & Gas/air-filled stomach herniated through a diaphragmatic defect \\
\hline Clinical Presentation & Acute dyspnea, chest pain, tachycardia \\
\hline Incidence & Rare, although incidence of congenital diaphragmatic hernia is 1 per 2,500-5000 live births \\
\hline Gender ratio & Slight female predominance \\
\hline Age predilection & Infants or adults, can occur at any age \\
\hline Risk factors & $\begin{array}{l}\text { Patients with diaphragmatic defects (either congenital or acquired i.e., following trauma, enlarging } \\
\text { hiatal hernia, status post diaphragm repair }\end{array}$ \\
\hline Treatment & Decompression via nasogastric tube followed by surgical repair of underlying diaphragmatic defect \\
\hline Prognosis & Excellent if promptly recognized and treated appropriately \\
\hline Imaging Findings & $\begin{array}{l}\text { Radiographs: large air-filled lucent structure in the left hemithorax with contralateral mediastinal } \\
\text { shift; absence of normal gastric bubble, poor visualization of the left hemidiaphragm } \\
\text { CT: similar findings to radiography however, cross-sectional imaging is helpful for identifying } \\
\text { communication between stomach and proximal small bowel }\end{array}$ \\
\hline
\end{tabular}

Table 1: Summary table of tension gastrothorax. 


\begin{tabular}{|l|l|l|l|}
\hline Diagnosis & General & X-ray & CT \\
\hline Tension pneumothorax & $\begin{array}{l}\text { Intrapleural accumulation of } \\
\text { air with ipsilateral lung } \\
\text { collapse }\end{array}$ & $\begin{array}{l}\text { Lucent hemithorax with } \\
\text { contralateral mediastinal shift, } \\
\text { can be left or right-sided }\end{array}$ & $\begin{array}{l}\text { Intact hemidiaphragm without } \\
\text { evidence of herniated } \\
\text { abdominal structures }\end{array}$ \\
\hline $\begin{array}{l}\text { Congenital } \\
\text { diaphragmatic hernia }\end{array}$ & $\begin{array}{l}\text { Diaphragmatic defect } \\
\text { detected on prenatal } \\
\text { ultrasound or shortly after } \\
\text { birth, typically } \\
\text { posterolaterally }\end{array}$ & $\begin{array}{l}\text { Indistinct diaphragm with } \\
\text { partial/full opacification of the } \\
\text { hemothorax (typically left- } \\
\text { sided); deviated lines and } \\
\text { tubes }\end{array}$ & $\begin{array}{l}\text { Rarely performed in neonatal } \\
\text { population }\end{array}$ \\
\hline
\end{tabular}

Table 2: Differential diagnosis table for tension gastrothorax radiographic mimics.

\section{Online access}

This publication is online available at: www.radiologycases.com/index.php/radiologycases/article/view/4144

$\mathrm{BPM}=$ beats per minute

$\mathrm{CT}=$ computed tomography

$\mathrm{mmHg}=$ millimeters of mercury

PACS $=$ picture archiving and communication system

\section{KEYWORDS}

Gastrothorax; Thoracic imaging; Emergency Radiology; Tension Pneumothorax; Diaphragmatic hernia; Lung ultrasound; X-ray; CT

\section{Peer discussion}

Discuss this manuscript in our protected discussion forum at: www.radiolopolis.com/forums/JRCR

\section{Interactivity}

This publication is available as an interactive article with scroll, window/level, magnify and more features. Available online at www.RadiologyCases.com

\section{Published by EduRad

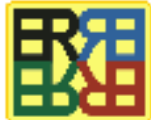

www.EduRad.org 